\title{
Configuration for Propellant Gauging in Satellites
}

\author{
Amit Lal* \\ Indian Institute of Science, Bangalore 560 012, India \\ and \\ B. N. Raghunandan \\ Indian Institute of Science, Bangalore 560 012, India \\ DOI: $10.2514 / 1.20709$
}

\begin{abstract}
Accurate propellant gauging is of prime importance in satellite industries. This paper explores the possibility of using a new propellant tank configuration, consisting of a truncated cone centrally mounted within a spherical propellant tank, to measure the amount of liquid propellant present within the tank. The liquid propellant present within the propellant tank orients itself in geometry by virtue of its dominant surface tension force in $0 \mathrm{~g}$ condition, which minimizes its total surface energy. This study reveals that the amount of liquid propellant present in the tank can thus be estimated by measuring the height of the propellant meniscus within the central cone. It is also observed that, for the proposed configuration, the precision of the estimated propellant fill-fraction increases towards the end of life of the spacecraft.
\end{abstract}

\section{Introduction}

A CCURATE determination of the amount of propellant present in a satellite toward its end of life is of prime importance in the determination of exact mission life and to plan a satellite replacement mission well in advance. Also, the annual revenue generated from a typical communication satellite operating at its full capacity is on the order of millions of dollars and, hence, premature removal of spacecraft from their orbits results in heavy losses.

Among the existing techniques, the bookkeeping method and the gas law method are extensively used. The bookkeeping method does not require additional instrumentation but the uncertainties associated with it are quite large; the uncertainty in the determination of mission life is on the order of $10 \%$ of total mission life. The propellant gauging system developed by Chobotov and Purohit [1] is better than the bookkeeping method, but an uncertainty analysis using Monte Carlo simulation suggests that the calculated propellant volume using the gas law method overestimates the actual amount of propellant present in the spacecraft [2]. Also, the extent of overprediction seems to increase toward the end of life of the spacecraft.

The major difficulty in the spacecraft propellant gauging stems from the fact that the propellant within the spacecraft tank is in a lowgravity environment. In this paper, an attempt is made to explore the possibility of exploiting the low-gravity condition to assist the propellant gauging. It was found that mounting a cone with its truncated vertex at the exit of the propellant tank, as shown in Fig. 1 , orients the propellant in a configuration favorable for measuring its content. The results of the study and the issues addressing the practical feasibility of the technique are discussed.

\section{Propellant Configuration Within the Propellant Tank}

In a low-gravity environment, the surface tension force of liquid propellant becomes the dominating force determining the final

\footnotetext{
Received 24 October 2005; revision received 30 August 2006; accepted for publication 10 September 2006. Copyright (C 2006 by the American Institute of Aeronautics and Astronautics, Inc. All rights reserved. Copies of this paper may be made for personal or internal use, on condition that the copier pay the $\$ 10.00$ per-copy fee to the Copyright Clearance Center, Inc., 222 Rosewood Drive, Danvers, MA 01923; include the code $\$ 10.00$ in correspondence with the CCC.

*Research Scholar, Department of Aerospace Engineering, Indian Institute of Science.

${ }^{\dagger}$ Professor, Department of Aerospace Engineering, Indian Institute of Science.
}

equilibrium configuration. The equilibrium configuration attained by the liquid propellant is the one that minimizes its total surface energy, which is given by [3]

$$
E=\sigma\left(A_{f}-A_{w} \cos \theta\right)
$$

where $\sigma$ is the interfacial liquid-vapor surface tension parameter and $A_{f}$ and $A_{w}$ are, respectively, the areas of liquid-vapor interface and of solid-liquid interface. As a consequence, a column of wetting liquid within a tapered tube tends to migrate toward the converging end of the tube. This behavior of liquid propellant has been made use of by mounting a cone with its truncated vertex at the exit of the propellant tank, as shown in Fig. 1 . The propellant is then confined to the conical and annular regions as indicated in Fig. 1 . If the propellant residing in the central cone increases, the free surface area of liquid propellant will increase, leading to increase in the surface energy of the liquid propellant. Such is the case with propellant residing in the annular region. Thus, the liquid propellant, both within the central cone and the annular region, stays as close to the cone's truncated vertex, conforming to an axisymmetric configuration and the liquid surface makes the same contact angle $\theta$ wherever it meets the tank surface.

\section{Propellant Configuration Within the Central Cone}

Because the equilibrium free surface attained by the liquid propellant is such that it has a constant mean curvature, the propellant's meniscus $F G F^{\prime}$ within the central cone (see Fig. 1) is part of a spherical surface.

Using the knowledge of solid geometry, the volume of the propellant contained in the central cone can be derived as

$$
V_{\text {cone }}=\frac{\pi r_{c}^{3}}{3}\left(\cot \alpha-\frac{2[1-\sin (\alpha+\theta)]}{\cos ^{3}(\alpha+\theta)}+\tan (\alpha+\theta)\right)
$$

where $\alpha, \theta$, and $r_{c}$ are the semicone angle of the central cone, contact angle of the liquid propellant with the tank material and the radial distance of point $F^{\prime}$ (which represents the line of contact of liquid propellant meniscus with the cone) from the axis of the cone, respectively. The free surface area of liquid propellant contained in the central cone is given by

$$
A_{f 1}=2 \pi r_{c}^{2}\left(\frac{1-\sin (\alpha+\theta)}{\cos ^{2}(\alpha+\theta)}\right)
$$

and the area of wetted surface formed by the liquid propellant in the central cone is given by 